\title{
Solutions of the higher-order Manakov-type continuous and discrete equations
}

\author{
A. Chowdury, A. Ankiewicz, and N. Akhmediev \\ Optical Sciences Group, Research School of Physics and Engineering, The Australian National University, Canberra ACT 0200, Australia
}

(Received 18 February 2014; published 7 July 2014)

\begin{abstract}
We derive exact and approximate localized solutions for the Manakov-type continuous and discrete equations. We establish the correspondence between the solutions of the coupled Ablowitz-Ladik equations and the solutions of the coupled higher-order Manakov equations.
\end{abstract}

DOI: 10.1103/PhysRevE.90.012902

PACS number(s): 05.45.Yv, 47.20.Ky, 42.81.Qb, 42.65.-k

\section{INTRODUCTION}

The nonlinear Schrödinger equation (NLSE) and a variety of its extensions have been among the most studied evolution equations. These equations and their solutions have been applied to explain experiments in many branches of physics for more than 50 years. In physics, the applications cover the fields of light-wave technology, plasma physics, water waves, photonics, nonlinear optics, optical waveguide arrays, Bose-Einstein condensation, and condensed-matter physics. In mathematics, NLSE research themes include integrability, various aspects of Riemann-Hilbert problems, stability theory of solitary waves, beam dynamics, chaos, and, very recently, extreme events.

In most cases, the NLSE deals with continuous systems, but to handle a physical system that is discrete by nature requires an appropriate discrete model. Ablowitz and Ladik first derived a discrete model that is an integrable discrete counterpart of the NLSE [1-4]. Like the NLSE itself, this discrete analog has a number of applications. A few examples can be mentioned in this regard: modeling a moving quasiparticle inside a periodic crystal and its interaction with lattice vibrations [5], temperature correlations of quantum spins [6], observing the dynamics of Bloch oscillations in an NLSE chain in the presence of static electric field [7] and soliton dynamics in discrete molecular chains [8]. Additional applications of the AL model include arrays of optical waveguides, electronic circuits, and the dynamics of nonlinear lattices [9-11]. A more detailed overview, applications, and relevant discretizations have been given in the review article in Ref. [12].

The Ablowitz- Ladik equation (ALE) and the NLSE are both integrable systems. Each can be solved using the well-known inverse scattering technique [13]. As the ALE is an integrable discretization of the NLSE, their solutions are also linked to each other. The most studied common solutions are soliton solutions on a zero background [1,14] or dark solitons [15], depending on the sign of the coefficients in the equation. Numerous techniques have been used to derive exact solutions of the ALE with reference to its related continuous (NLSE) partner solutions [16-18]. The explicit connection between the exact solutions of the ALE and NLSE has been given in a recently published paper [19]. The latest new solutions of the ALE are rogue waves. Their nature, mathematical interpretation, and occurrences have been discussed in Refs. [20,21]. The phenomena of modulation instability, Fermi-Pasta-Ulam recurrence, rogue waves, nonlinear phase shift, and the corresponding exact solutions of the Ablowitz-Ladik equation have been discussed in [19]. A convenient approach to first-order exact solutions of the ALE has been presented in [22]. The second-order discrete rogue wave solution of the Ablowitz-Ladik equation has been given in [23].

Our aim here is to find solutions of coupled AL equations by extending a Manakov model to a discrete case. A basic twocomponent "tanh" and "sech" solution for discrete coupled AL equations was derived in Ref. [19]. The coupled set of AL equations for the defocusing case has been solved in terms of Lamé and Chebyshev polynomials in [24,25]. Our present endeavor is to find different solutions of the AL equations using ideas from Ref. [26], where an $N$-coupled set of focusing NLSEs has been solved in terms of Legendre polynomials. In the continuous limit, these solutions converge to the solutions of the set of Manakov equations in both the focusing and defocusing forms.

\section{DISCRETE AND CONTINUOUS COUPLED EQUATIONS}

The standard normalized integrable AL equation can be written as

$$
\begin{gathered}
i \frac{d \psi_{n}^{(1)}}{d t}+\frac{1}{2 h^{2}}\left(\psi_{n-1}^{(1)}+\psi_{n+1}^{(1)}-2 \psi_{n}^{(1)}\right) \\
+\frac{\alpha}{2}\left(\psi_{n-1}^{(1)}+\psi_{n+1}^{(1)}\right)\left|\psi_{n}^{(1)}\right|^{2}=0
\end{gathered}
$$

where $n=\cdots-2,-1,0,1,2, \ldots$ is the discrete variable and $h$ is the discretization parameter. The equation in this form includes defocusing ( $\alpha=-1$, where the nonlinear response decreases with increasing intensity) and focusing $(\alpha=+1$, where the nonlinear response increases with increasing intensity) cases. Equation (1) is a second-order finite-difference approximation of the standard NLSE [Eq. (2.1) of [27]]:

$$
i \frac{\partial \psi}{\partial t}+\frac{1}{2} \psi_{x x}+\alpha|\psi|^{2} \psi=0
$$

This particular integrable discretization has been discussed in $[19,22,28,29]$. Complex functions $\psi_{n}(t)$ and $\psi(t, x)$ in Eqs. (1) and (2) are the wave functions for the discrete and continuous cases, respectively. If we set $n=x / h$, the limit of $h \rightarrow 0$ reduces the discrete ALE to the continuous NLSE. For both ALE and NLSE, $\alpha=1$ means focusing and $\alpha=-1$ means a defocusing system. A detailed and systematic procedure for lining up the ALE and NLSE and their corresponding solutions by using standard transformations and scaling of the $t$ and $h$ variables was given in [19].

Equations (1) and (2), when applied to light-wave technology, usually deal with unidirectional polarized light consisting 
of a single component. More complicated systems can be described by the multicomponent NLSEs and ALEs. The partially coherent solitons (PCSs) are one example where multicomponent coupled NLSEs are needed to provide a detailed understanding of the formation of PCSs, their shape, and collision properties [30-32]. The standard generalized coupled ALE system, which is basically a discretized set of NLSEs, can be written as

$$
\begin{aligned}
& i \frac{d \psi_{n}^{(1)}}{d t}+\frac{1}{2 h^{2}}\left(\psi_{n-1}^{(1)}+\psi_{n+1}^{(1)}-2 \psi_{n}^{(1)}\right) \\
& \quad+\frac{\alpha}{2}\left(\psi_{n-1}^{(1)}+\psi_{n+1}^{(1)}\right)\left(\left|\psi_{n}^{(1)}\right|^{2}+\left|\psi_{n}^{(2)}\right|^{2}\right)=0, \\
& i \frac{d \psi_{n}^{(2)}}{d t}+\frac{1}{2 h^{2}}\left(\psi_{n-1}^{(2)}+\psi_{n+1}^{(2)}-2 \psi_{n}^{(2)}\right) \\
& \quad+\frac{\alpha}{2}\left(\psi_{n-1}^{(2)}+\psi_{n+1}^{(2)}\right)\left(\left|\psi_{n}^{(1)}\right|^{2}+\left|\psi_{n}^{(2)}\right|^{2}\right)=0 .
\end{aligned}
$$

In the case of just two sets, we can call them "discretized Manakov equations." Indeed, in the limit $n h=x$ and $h \rightarrow 0$, the above system reduces to the continuous coupled Manakov equations with two components [19], which, for the focusing case $(\alpha=1)$, can be written as

$$
\begin{aligned}
& i \frac{\partial \psi^{(1)}}{\partial t}+\frac{1}{2} \psi_{x x}^{(1)}+\left(\left|\psi^{(1)}\right|^{2}+\left|\psi^{(2)}\right|^{2}\right) \psi^{(1)}=0, \\
& i \frac{\partial \psi^{(2)}}{\partial t}+\frac{1}{2} \psi_{x x}^{(2)}+\left(\left|\psi^{(1)}\right|^{2}+\left|\psi^{(2)}\right|^{2}\right) \psi^{(2)}=0 .
\end{aligned}
$$

Higher-order sets of coupled equations describe so-called incoherent solitons. The concept of incoherent solitons or random-phase nonlinear waves was introduced by Hasegawa [32-35] more than 35 years ago. The experiment carried out by Mitchell et al. [36] extended this concept to the case of spatial solitons. This experiment confirmed that incoherent solitons do exist and are observable. Photorefractive materials turned out to be ideal for experimental observations of self-trapping of partially coherent light, due to their slow response time [37] and low optical power requirement for the development of strong nonlinear effects [38,39]. Christodoulides et al. $[40,41]$ have shown that incoherent self-trapped light inside a photorefractive material can be represented by an infinite set of coupled nonlinear Schrödinger equations (NLSs) [40]. The theory of incoherent solitons within nonlinear Kerr-like media has been developed in [32]. This theory allowed researchers to study the shapes of incoherent solitons and their changes after collisions. They are described by multiparameter families of solutions, leading to arbitrary shapes of solitons in the limit of complete incoherence [32]. We will provide approximate solutions to Eq. (3) in Sec. VI.

The stationary solutions for incoherent solitons, their dynamics, and collisions have also been analyzed in [42-45]. A comprehensive description of partially coherent solitons has been given in [30]. This work, which follows [46], elucidates soliton shapes and their collisions in terms of exact analytic solutions. It was found that for equally spaced eigenvalues, a symmetric solution of $M$ coupled NLS equations can be derived in terms of associated Legendre functions, representing symmetric partially coherent solitons on zero background. They are based on the modes of the "sech-squared" waveguide
[31]. Here, the symmetry and "sech-squared" guide reduce the solution families from multiparameter to single-parameter solutions [45]. As the amplitude of partially coherent solitons provides another parameter to the solution, finally a twoparameter symmetric solution has been derived in [26] for arbitrary $M$ coupled NLSEs in terms of associated Legendre functions; it is on a finite background. Here, we generalize [26] presenting solutions for both defocusing $(\alpha=-1)$ and focusing $(\alpha=1)$ cases.

\section{SELF-TRAPPED INCOHERENT SOLITONS: DEFOCUSING $(\alpha=-1)$ AND FOCUSING $(\alpha=1)$ CASES}

Propagation of an $M$-component self-trapped mutually incoherent soliton set in photorefractive media can be expressed in terms of the solution of a set of focusing NLSEs given by

$$
i \frac{\partial \psi_{j}(x, t)}{\partial t}+\frac{1}{2} \frac{\partial^{2} \psi_{j}(x, t)}{\partial x^{2}}+\alpha \delta n \psi_{j}(x, t)=0,
$$

where $\psi_{j}(x, t)$ is the $j$ th component of the beam $(j=$ $1, \ldots, M), x$ is the spatial dimension, $t$ is time variable or propagation direction, and $\alpha$ is a coefficient denoting the strength of the nonlinearity. When $\alpha=1$, Eq. (6) is the focusing NLSE, and when $\alpha=-1$ it becomes the defocusing NLSE. The total refractive index change is

$$
\delta n=\sum_{j=1}^{M}\left|\psi_{j}\right|^{2}
$$

We are looking for solutions of Eq. (6) with $\alpha= \pm 1$ in the form

$$
\psi_{j}(x, t)=u_{j}(x) e^{i k_{j} t},
$$

where $j=1, \ldots, M$. Propagation constants $k_{j}$ are different for each $j$; this makes the components mutually incoherent. This ansatz reduces Eq. (6) to the set of ordinary differential equations (ODEs):

$$
u_{j}^{\prime \prime}(x)+2 \alpha u_{j}(x) \sum_{i=1}^{M} u_{i}^{2}(x)=2 k_{j} u_{j}(x)
$$

for $j=1, \ldots, M$. The two- and three-component cases of Eq. (6) have been considered in [47]. In particular, Hioe [47] derived a dark-bright soliton solution of Eq. (6). The higher-order solution sets for arbitrary $M$ have been found in [26]. However, only the focusing case, $\alpha=1$, has been considered so far. The solutions comprise families with the eigenvalues arranged in decreasing order. We now generalize these solutions, allowing both positive and negative coefficients, i.e., $\alpha= \pm 1$. Even for positive $\alpha=+1$, we present these solutions in a simpler form that involves two arbitrary parameters.

Namely, the solution of Eq. (9) can be presented in terms of associated Legendre polynomials with the total intensity in Eq. (9) set to

$$
\begin{aligned}
\delta n= & \sum_{i=1}^{M} u_{i}^{2}(x)=b_{1}(M) a_{1} \\
& -\alpha(M-1) p^{2}\left[M-1-\frac{M}{2} \operatorname{sech}^{2}(p x)\right],
\end{aligned}
$$


where $b_{1}(M)=\frac{1}{30}\left(4+15 M-M^{2}\right)$ and $a_{1}$ and $p$ are two arbitrary parameters. Clearly, the first term in (10) has to be positive, i.e., we need $b_{1}(M) a_{1}>0$. If we assume that $M$ is an arbitrary integer, change the variable $y=p x$, and rearrange Eq. (9) to the form

$$
p^{2} \frac{d^{2} u_{j}}{d y^{2}}+2 \alpha \delta n u_{j}=2 k_{j} u_{j},
$$

then the solution of (11) can be expressed in terms of associated Legendre polynomials:

$$
u_{j}(y)= \pm \sqrt{c_{M, j}} P_{M-1}^{M-j} \tanh (y)
$$

for $j=1, \ldots, M$. The coefficients of the polynomial $c_{M, j}$ must be chosen in such a way that (12) is a solution of (11). This happens when

$$
\begin{aligned}
c_{M, j}= & \frac{2(j-1) !}{(2 M-j-2) !\left(\delta_{j, M}+1\right)} \\
& \times\left[\frac{a_{1} b_{1}(M)}{2 M-j-1}-\alpha(j-1) p^{2}\right],
\end{aligned}
$$

where the Kronecker delta function is $\delta_{j, M}=0$ for $M \neq j$ and 1 for $M=j$. The propagation constants in (11) that correspond to the above solutions are

$$
k_{j}=\alpha a_{1} b_{1}(M)-p^{2}\left[(M-1)^{2}-\frac{1}{2}(M-j)^{2}\right] .
$$

The expressions under the square root in (12) must not be negative, starting with the higher-order one, $j=M$, i.e., $c_{M, M} \geqslant 0$. This means that

$$
a_{1}\left(4+15 M-M^{2}\right) \geqslant 30 \alpha(M-1)^{2} p^{2} .
$$

This ensures that all lower-order coefficients with $j=$ $1,2, \ldots, M-1$ are also non-negative $c_{M, j} \geqslant 0$. For example, if $\alpha=+1$ and $M \geqslant 16$, then $b_{1}(M)<0$ and we need to choose $a_{1}<0$.

The number of components, $M$, controls the overall profile of the solution. For the lowest-order nontrivial case $M=2$, the general solution is

$$
\begin{aligned}
& u_{1}(x)=-\sqrt{a_{1}} \operatorname{sech}(p x), \\
& u_{2}(x)=\sqrt{a_{1}-p^{2} \alpha} \tanh (p x),
\end{aligned}
$$

while the total intensity $\delta n$ is given by

$$
\sum_{i=1}^{2} u_{i}^{2}(x)=a_{1}-\alpha p^{2} \tanh ^{2}(p x)
$$

For $M=3$, the general solution can be written as

$$
\begin{aligned}
& u_{1}(x)=\sqrt{a_{1}} \operatorname{sech}^{2}(p x), \\
& u_{2}(x)=-\sqrt{4 a_{1}-9 p^{2} \alpha} \tanh (p x) \operatorname{sech}(p x), \\
& u_{3}(x)=\frac{1}{\sqrt{3}} \sqrt{a_{1}-3 p^{2} \alpha} \tanh (p x)\left[3 \tanh ^{2}(p x)-1\right],
\end{aligned}
$$

while the intensity for this case is

$$
\sum_{i=1}^{3} u_{i}^{2}(x)=\frac{4 a_{1}}{3}-\alpha p^{2}\left[4-3 \operatorname{sech}^{2}(p x)\right] .
$$

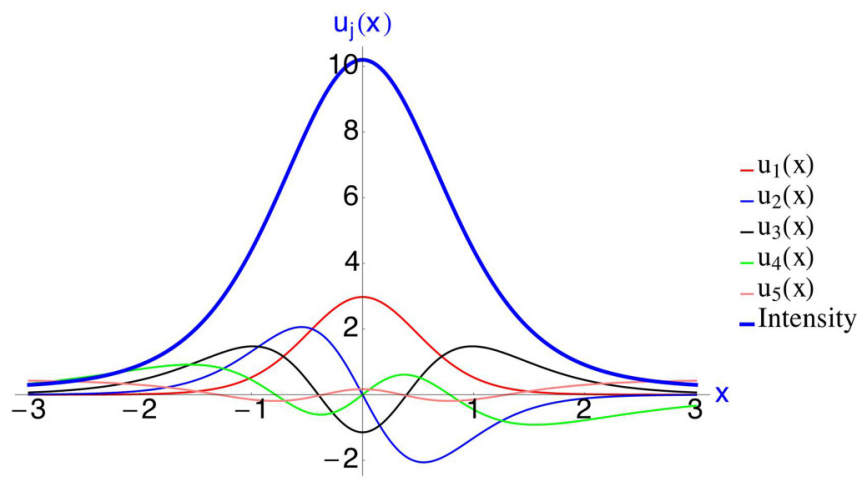

(a)

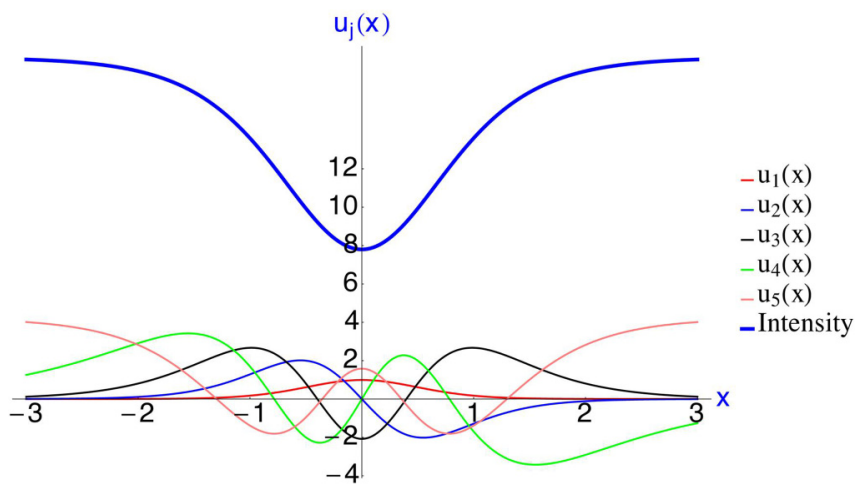

(b)

FIG. 1. (Color online) An example of an incoherent soliton for $M=5$ in the (a) focusing $(\alpha=+1)$ and (b) defocusing $(\alpha=-1)$ cases. Parameters are chosen as follows: for the focusing case $a_{1}=9, p=1$ while for the defocusing case $a_{1}=1, p=1$. The overall intensity profiles $\delta n$ are shown by the solid (blue) curves. The five modes of the solution are shown by the thin colored curves with the legends presented on the right-hand side of each figure.

The solutions for higher values of $M$ can be written in a similar way. They all contain two arbitrary real parameters $a_{1}$ and $p$. A particular soliton with $M=5$ is illustrated in Fig. 1(b) for both focusing and defocusing cases.

\section{REDUCTION TO THE ZERO-BACKGROUND SET OF ORDER $M-1$}

In the general case, the solution has a nonzero background. The background level of the whole intensity is defined by the last component of the solution in Eq. (13). The background intensity can be set to zero if we set the last component coefficient, $c_{M, M}$, to be zero, i.e.,

$$
a_{1} b_{1}(M)-\alpha p^{2}(M-1)^{2}=0 .
$$

This can only be done for the focusing case $(\alpha=1)$, as $\sqrt{c_{M, j}}$ remains real for all $M$ whether $b_{1}(M)$ is positive or negative. By solving (20) for the focusing case, we get

$$
a_{1}=\frac{30(M-1)^{2} p^{2}}{4+15 M-M^{2}} .
$$

Inserting this value of $a_{1}$ into Eqs. (12) and (13), we obtain each real-valued component $u_{M, j}$ forming the solution set. 
Then, the last component is zero and so the overall background is also zero. For example, in order to obtain the solution with zero background for $M=3$, we find $a_{1}=3 p^{2}$ from Eq. (21) and substitute it into Eq. (13). The result is

$$
\begin{aligned}
& u_{1}(x)=\sqrt{3} p \operatorname{sech}^{2}(p x) \\
& u_{2}(x)=\sqrt{3} p \tanh (p x) \operatorname{sech}(p x)
\end{aligned}
$$

Clearly, this is the solution set from [30] given by Eqs. (29) and (30). The coefficient of the $j$ th component of the zero background solution for any $M$ is then given by $\sqrt{c_{M, j}}$ from Eq. (13). It now takes this simpler form,

$$
c_{M, j}=2 \frac{(j-1) !(M-j)^{2}}{(2 M-j-1) !} p^{2},
$$

where $j=1,2,3, \ldots, M$. This expression is always $\geqslant 0$, as required. The propagation constant (14) of the $j$ th component of the solution set is now given by

$$
k_{j}=\frac{1}{2}(M-j)^{2} p^{2} .
$$

For any $M$, the total intensity is given by

$$
\sum_{j=1}^{M} u_{j}^{2}=\frac{M}{2}(M-1) p^{2} \operatorname{sech}^{2}(p x) .
$$

For example, the zero-background solution set for $M=6$ and $\alpha=1$ is

$$
\begin{aligned}
& u_{1}(x)=-\frac{15}{4} \sqrt{7} p \operatorname{sech}^{5}(p x) \\
& u_{2}(x)=\frac{3}{2} p \sqrt{35} \operatorname{sech}^{4}(p x) \tanh (p x) \\
& u_{3}(x)=-\frac{3}{8} \sqrt{\frac{35}{2}} p \operatorname{sech}(p x) \operatorname{sech}^{4}(p x)[4 \cosh (2 p x)-5] \\
& u_{4}(x)=\frac{1}{4} \sqrt{105} p \operatorname{sech}^{5}(p x)[\sinh (3 p x)-5 \sinh (p x)]
\end{aligned}
$$

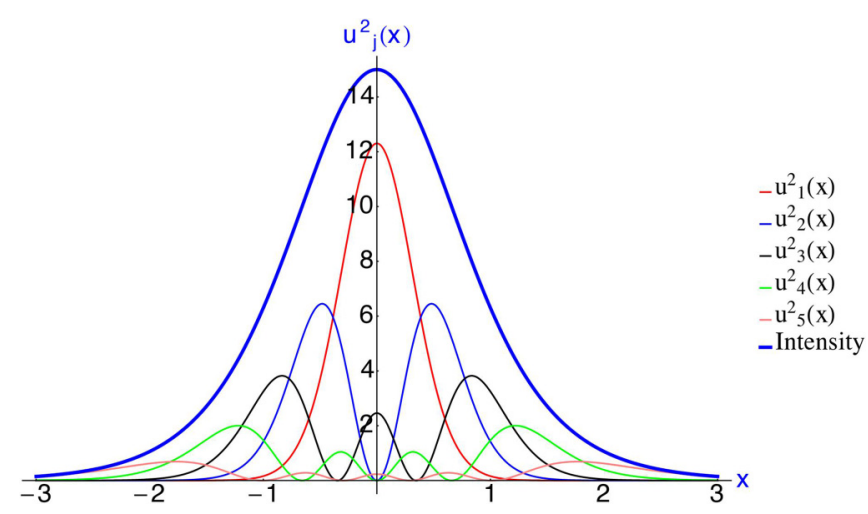

FIG. 2. (Color online) An example of an incoherent soliton for $M=6$ in the focusing $(\alpha=+1)$ case. The parameter $p=1$. The overall intensity profile $\delta n$ is shown by the solid (blue) curve. The individual intensities of the components of the solution are shown by the thin colored curves with the legends presented on the right-hand side of the figure.

$$
\begin{aligned}
u_{5}(x)= & -\frac{1}{8} \sqrt{15} p \operatorname{sech}(p x)\left[21 \tanh ^{4}(p x)\right. \\
& \left.-14 \tanh ^{2}(p x)+1\right] \\
u_{6}(x)= & 0 .
\end{aligned}
$$

This solution is presented in Fig. 2.

The total intensity background (i.e., $\delta n$ at $x= \pm \infty$ ) equals the background intensity of the last component, since all other components approach zero as $x=\rightarrow \pm \infty$. So, setting the background part of the total intensity to zero by using the condition (21) also makes the last component of the solution set zero and provides the full set with $M-1$ zero-background components.

\section{HIGHER-ORDER SOLUTIONS OF THE ABLOWITZ-LADIK FOCUSING EQUATIONS}

The Ablowitz-Ladik model $(h=1)$ presented in $[24,25]$ is slightly different from the set of equations (3) considered here. A solution of this coupled defocusing AL set has been expressed in [25] using Chebyshev polynomials of the first and second kinds and making a connection with Lamé polynomials. Generally, the solution of Eq. (3) for arbitrary $h, p$ can be written as a pair:

$$
\begin{gathered}
\psi_{n}^{(1)}=\frac{1}{h} F(h n p) e^{i t / h^{2}}, \\
\psi_{n}^{(2)}=\frac{1}{h} \sqrt{1-F^{2}(h n p)} e^{i t / h^{2}}
\end{gathered}
$$

for an arbitrary real function $F(h n p)$. The Chebyshev polynomials fit this form. However, any other function can also be used.

The solution of the order $M=3$ admits two arbitrary functions:

$$
\begin{aligned}
\psi_{n}^{(1)} & =\frac{1}{h} F(h n p) e^{i t / h^{2}}, \\
\psi_{n}^{(2)} & =\frac{1}{h} G(h n p) e^{i t / h^{2}}, \\
\psi_{n}^{(3)} & =\frac{1}{h} \sqrt{1-F^{2}(h n p)-G^{2}(h n p)} e^{i t / h^{2}} .
\end{aligned}
$$

These arbitrary functions are $F(h n p), G(h n p)$.

The common property of these solutions is that the total intensity is always 1 . Clearly we can have a solution with an arbitrary number of components of the general form

$$
\psi_{n}^{(j)}=\frac{1}{h} F_{j}(h n p) e^{i t / h^{2}},
$$

where $j=1,2, \ldots, k-1$, and with the last component

$$
\psi_{n}^{(k)}=\frac{1}{h} \sqrt{1-\sum_{j=1}^{k-1} F_{j}^{2}(h n p)} e^{i t / h^{2}} .
$$

The phase factor $e^{i t / h^{2}}$ shows that we cannot have a continuous limit $h \rightarrow 0$ for this defocusing case. 


\section{EXPANSION}

In the continuous example (6), solutions of the focusing case, when $\alpha=1$, differ significantly from the defocusing one, $\alpha=-1$. In the work [30], exact solutions have been given in terms of Legendre polynomials for $N$ coupled Manakov equations. This solution describes partially coherent solitons (PCSs) with zero background in a Kerr medium. Now, if we turn to the discretized version of Eq. (6) from [30] for $N=2$, we obtain a coupled AL system, Eq. (3).

The recent work [19] makes it clear that there is a correspondence between the solutions of the integrable ALE system and the NLSE. Based on this newly established principle, we assume that, for the focusing case, there may be some solution set for the ALE system which has its $h \rightarrow 0$ limit in Eqs. (29) and (30) of [30], given as Eq. (22) in this present paper:

$$
\begin{aligned}
& \psi^{(1)}=\sqrt{3} \operatorname{sech}^{2}(x) \exp (2 i t), \\
& \psi^{(2)}=\sqrt{3} \operatorname{sech}(x) \tanh (x) \exp (i t / 2),
\end{aligned}
$$

where $x$ is the transverse spatial variable, $t$ is the propagation variable, and we have taken $k_{1}=2$ and $k_{2}=1 / 2$.

In the Introduction, we mentioned general optical and electrical applications of the ALE. In Sec. II, we explained the application of this Manakov-type formalism to incoherent light propagation in photorefractive media. Another physical application can be a set of two arrays of optical waveguides located one above the other. Then the overall solution has two component sets, one for the lower array and one for the upper. For sufficiently close arrays, there will be coupling between them. As another example, this particular coupled ALE set can be used to model sophisticated soliton dynamics and energy exchange between two ladder lattices [48-50] in the presence of intersite impurities. This application may require an investigation of energy transfer in the "exciton-vibron" system [51].

Now we represent the corresponding coupled ALE solution of Eq. (3) with an associated multiplying function as

$$
\begin{aligned}
& \Psi_{n}^{(1)}=\sqrt{3} u_{1}(n, h) \operatorname{sech}^{2}(n h) \exp \left(i k_{1} t\right), \\
& \Psi_{n}^{(2)}=\sqrt{3} u_{2}(n, h) \operatorname{sech}(n h) \tanh (n h) \exp \left(i k_{2} t\right) .
\end{aligned}
$$

Here the unknown functions $u_{1}(n, h), u_{2}(n, h)$, and the constants in the exponent, $k_{1}$ and $k_{2}$, have to be determined so that the set will solve Eq. (3). So we require $u_{1}(n, 0)=1$, $u_{2}(n, 0)=1$. When $h=0$, we need $k_{1}=2$ and $k_{2}=1 / 2$. We expand the functions as

$$
\begin{aligned}
& u_{1}(n, h)=1+h^{2} y_{1}(n)+h^{4} y_{2}(n)+h^{6} y_{3}(n)+\cdots, \\
& u_{2}(n, h)=1+h^{2} z_{1}(n)+h^{4} z_{2}(n)+h^{6} z_{3}(n)+\cdots,
\end{aligned}
$$

and the constants as

$$
\begin{aligned}
& k_{1}=2+h^{2} a_{1}+h^{4} a_{2}+h^{6} a_{3}+h^{8} a_{4}+\cdots, \\
& k_{2}=1 / 2+h^{2} b_{1}+h^{4} b_{2}+h^{6} b_{3}+h^{8} b_{4}+\cdots .
\end{aligned}
$$

Now, substituting Eqs. (31) and (32) into Eq. (3), taking an expansion in terms of $h$ up to high order, and solving for the undetermined coefficients, we find that $k_{1}$ and $k_{2}$ turn out to be

$$
\begin{aligned}
& k_{1}=\frac{2 \sinh ^{2}(h)}{h^{2}}, \\
& k_{2}=\frac{2 \sinh ^{2}\left(\frac{h}{2}\right)}{h^{2}} .
\end{aligned}
$$

\section{APPROXIMATE SOLUTION}

Generally, the functions $u_{1}(n, h)$ and $u_{2}(n, h)$ are infinite even-order polynomials in $h$ and $\tanh (n h)$. The contribution terms become smaller with increasing order of $h$, so we can ignore terms that are of very high order in $h$. These infinite polynomials can be approximated in a simpler form in terms of a finite specific function, which we call $D(n)$. We find that a convenient, yet simple, approximation to the solution of the pair of ALEs can be written in terms of this function, $D(n)=H /(1-H)$, where $H=h^{2} \tanh ^{2}(h n)$ :

$$
\begin{aligned}
& \frac{u_{1}(n, h)}{1+D(n)} \approx \frac{3}{3+2 h^{2} D(n)}+f(h)+D(n) u(h), \\
& \frac{u_{2}(n, h)}{1+D(n)} \approx \frac{3}{3+2 h^{2} D(n)}+g(h)+D(n) y(h) .
\end{aligned}
$$

The cumbersome functions $f(h), u(h), g(h)$, and $y(h)$, which are polynomials in $h$, are given in the Appendix. Thus, we have the complete form of the approximate solution:

$$
\begin{aligned}
& \Psi_{n}^{(1)} \approx \sqrt{3} u_{1}(n, h) \operatorname{sech}^{2}(h n) \exp \left(\frac{2 i t \sinh ^{2}(h)}{h^{2}}\right), \\
& \Psi_{n}^{(2)} \approx \sqrt{3} u_{2}(n, h) \tanh (h n) \operatorname{sech}(h n) \exp \left(\frac{2 i t \sinh ^{2}\left(\frac{h}{2}\right)}{h^{2}}\right) .
\end{aligned}
$$

The factors $u_{1}(n, h)$ and $u_{2}(n, h)$ can be approximated with simple functions,

$$
\begin{aligned}
& u_{1}(n, h) \approx \cosh ^{2 / 3}(h) \cosh ^{\left(h^{2}\right)}(n h), \\
& u_{2}(n, h) \approx \cosh ^{\left(h^{2} / 2\right)}(n h) .
\end{aligned}
$$

This means that

$$
\begin{aligned}
& \Psi_{n}^{(1)} \approx \sqrt{3} \cosh ^{2 / 3}(h) \operatorname{sech}^{\left(2-h^{2}\right)}(n h) \exp \left(i k_{1} t\right), \\
& \Psi_{n}^{(2)} \approx \sqrt{3} \tanh (n h) \operatorname{sech}^{\left(1-h^{2} / 2\right)}(n h) \exp \left(i k_{2} t\right),
\end{aligned}
$$

with $k_{1}, k_{2}$ given by Eqs. (33) and (34) above. Of course, Eqs. (38) and (39) provide the shapes but are not as accurate as Eqs. (35) and (36). The functions (39) are approximations of Eq. (37).

The solutions are accurate within the limits $0<h<\frac{3}{4}$. The expansion is correct up to the order $h^{13}$. In Fig. 3, the perturbation curves have their minima slightly above and below 1 , respectively. In effect, these alter the amplitude of the total solution to some extent. For example, $\Psi_{n}^{(1)}$ increases from an unperturbed value 1.73 to 2.12 , while the other component $\Psi_{n}^{(2)}$ maximum increases from an unperturbed value 0.87 to 1 . Another important observation is that if we want to increase the accuracy of the solution, we have to take into account the higher-order terms in $h$. However, we find that there is very 


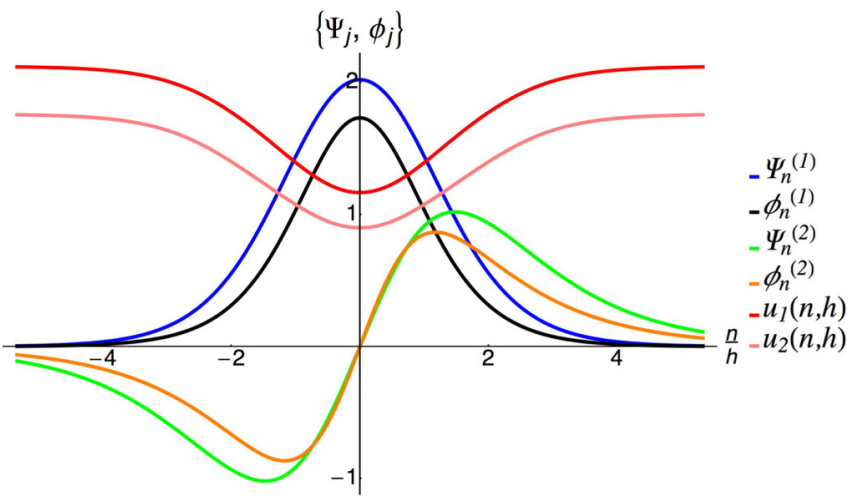

FIG. 3. (Color online) Profiles of the solution for coupled AL with $h=0.75$. Perturbed and unperturbed $(h \rightarrow 0)$ forms of the solution are presented separately with different color profiles. Here, real functions $\Psi_{n}^{(1)}$ and $\Psi_{n}^{(2)}$ (with $t=0$ ), shown with blue and green, represent the total solution. From the figure, the scaling of the transverse variable $n$ with interlattice spacing $h$ is also clear. For clarity, we have taken the total range between $n / h=-4$ and 4 . The full solution profile deviates from that of the unperturbed $(h \rightarrow 0)$ solution profiles $\phi_{n}^{(1)}$ and $\phi_{n}^{(2)}$ (with $t=0$ ), which are shown in black and orange, respectively. Red $\left[u_{1}(n, h)\right]$ and pink $\left[u_{2}(n, h)\right]$ curves show the perturbation factors (38) applied to the total solution.

little contribution to the solution profile from high-order terms in $h$. As an example, the contribution is of the order of $10^{-6}$ for $h=0.75$ for terms of the order of $h^{13}$.

Now, if we set $n=x / h$ and take the limit $h \rightarrow 0$, the perturbed functions $u_{1}(n, h)$ and $u_{2}(n, h)$ both reduce to 1 , and for the phase factors in the solution, we have $k_{1}=\frac{2 \sinh ^{2}(h)}{h^{2}} \rightarrow$ 2 and $k_{2}=\frac{2 \sinh ^{2}\left(\frac{h}{2}\right)}{h^{2}} \rightarrow \frac{1}{2}$. Hence, the approximate solution of Eq. (37) will approach the solution set for the continuous coupled NLSEs, which is the coupled Manakov set. In the limit $h \rightarrow 0$, it reduces to the solution given in Eq. (29). Our plots of the solutions give an idea of how much the solution of the coupled ALE set deviates from that of the continuous Manakov set for small value of $h$.

\section{NUMERICS AND APPROXIMATIONS}

To justify the analytical theory of our solution, we used numerical calculations. In the numerics, the solution represented by Eq. (37) has been used as the initial condition in the beam propagation scheme. The propagation was coupled and consistent, and the results have been verified from different prospectives. Values for the variable $n$, which is the discrete lattice point, are chosen between -12 and 12. The distance between two consecutive lattice points has been scaled with the interlattice distance variable, $h$, so that we can see how each component of the solution actually scales with $h$. The pattern evolves with $t$, which is the propagation variable, ranging from 0 to 100 . We have checked that the propagation profile is intact for even longer propagation distances. If we look at the propagation profile of the solution in Figs. 4(a) and 4 (b) with $h=0.75$, then for both components of the solution, it becomes obvious that the soliton profiles are intact, as predicted in the theoretical explanation above. The lower profiles in Figs. 4(c) and 4(d) show the same solution profile (a)

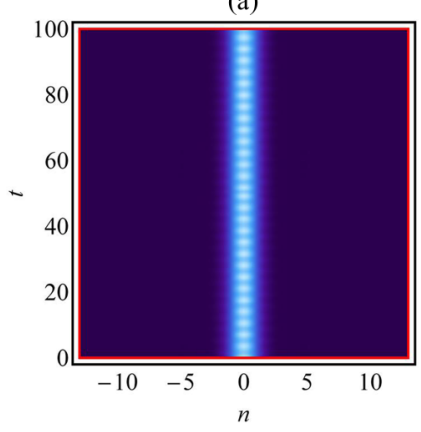

(c)

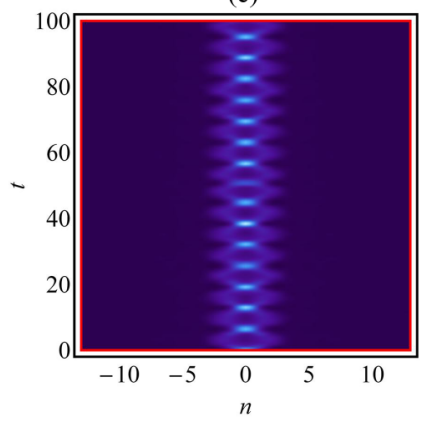

(b)

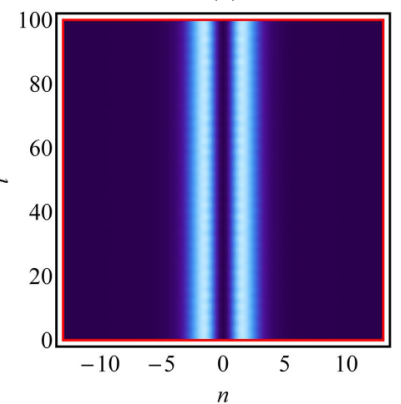

(d)

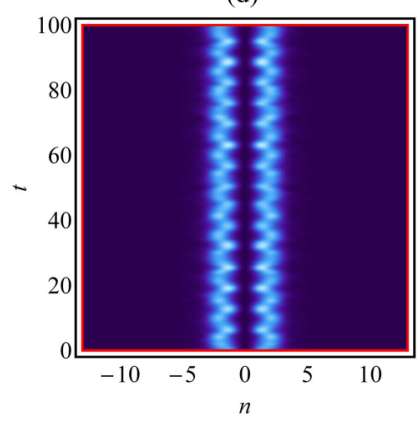

FIG. 4. (Color online) Soliton propagation for $\left|\Psi_{1}(n)\right|$ and $\left|\Psi_{2}(n)\right|$. Here at the top, (a) and (b) show the first and second components of the solution for $h=0.75$, while (c) and (d) show the propagation for $h=0.85$.

with $h=0.85$. In that case, we observe that solitons actually are not in their steady-state condition, and they start to scatter with propagation. This verifies that our approximate solutions are valid up to $h=0.75$.

To verify the analytic approximate solution of the coupled AL set, we have extended our numerical investigation further. The phase parameters of the solution [see Eq. (19)], viz., $k_{1}$ and $k_{2}$, for components $\Psi_{n}^{(1)}$ and $\Psi_{n}^{(2)}$, respectively, are functions of $h$ only. We have compared the numerically determined variations of $k_{1}, k_{2}$ with the analytical expressions $k_{1}=\frac{2 \sinh ^{2}(h)}{h^{2}}$ and $k_{2}=\frac{2 \sinh ^{2}\left(\frac{h}{2}\right)}{h^{2}}$. In principle, $k_{1}$ and $k_{2}$ should be independent of $n$ and $t$. Now, if we look at Fig. 5(a), the red line represents the analytic expression for the phase of the first component, $k_{1}$, as a function of $h$ and the blue dots are the numeric values. It can be seen that the blue dots start to deviate from the analytic line around $h=0.75$, which is our estimated maximum value for which our coupled approximate solution is valid. In the same way, Fig. 5(b) shows the phase

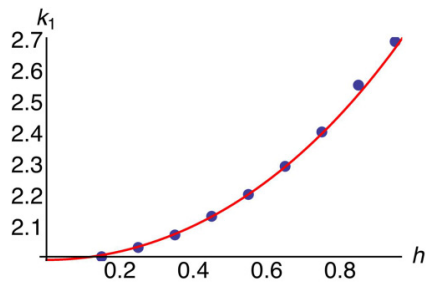

(a)

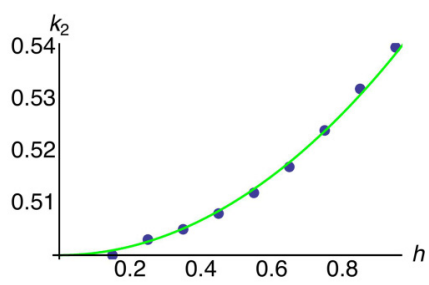

(b)
FIG. 5. (Color online) Comparison of phase parameters (a) $k_{1}$ and (b) $k_{2}$, found numerically (dots) with their analytic forms (curves). 
profile for the second component, $k_{2}$, where the blue dots start to deviate from the analytic line at $h=0.75$.

\section{CONCLUSION}

In this work, we have shown that the solution of the coupled Manakov system, with any number of modes, can be expressed in terms of Legendre polynomials. This can be done for both focusing and defocusing systems. Remarkably, the rational coefficients of the polynomials can be expressed using just a single function in each case.

We have also derived an approximate solution for the twocomponent ALE. We have shown that, in the limit $h \rightarrow 0$, the solution of the two-component ALE becomes the solution of the two-component Manakov system. By using numerical simulations, we have verified that the approximate solution is valid for a wide range of the parameter values, viz., $0<h<$ 0.75 .

The main idea behind this work is that, since the ALE reduces to the NLSE in the limit $h \rightarrow 0$, the solutions should also show the same correspondence. For various cases, we have seen that this particular procedure is useful, following on from our previous work [19]. Using this analogy here, we have worked to find a related solution of the Manakov system of the coupled ALEs, and we have provided an approximate solution. It remains a computational challenge to find a systematic way to derive exact solutions for infinite-component coupled ALEs, and we look forward to a resolution of this challenge in the future.

\section{ACKNOWLEDGMENTS}

The authors acknowledge the support of the ARC (Discovery Project DP140100265). N.A. and A.A. acknowledge the support of the Volkswagen Stiftung, while A.C. is grateful for support through an Endeavour Fellowship.

\section{APPENDIX}

The functions used in Eqs. (35) and (36) are

$$
\begin{aligned}
& f(h)=\frac{2 h^{2}}{3 !}-2 \frac{(2 h)^{4}}{3 \times 5 !}+\frac{61(2 h)^{6}}{3 \times 4 \times 7 !}-\frac{59(2 h)^{8}}{9 !} \\
& +3 \times 19 \times 431 \frac{(2 h)^{10}}{4 \times 11 !}+3^{5} \frac{119923 h^{12}}{112 \times 13 !} \\
& +2^{8} \frac{32552473157 h^{14}}{21 \times 15 !}+\cdots, \\
& u(h)=8 h^{4}\left(\frac{7}{3 \times 5 !}-2 \frac{11 h^{2}}{3 \times 7 !}\right. \\
& -7 \times 24 \frac{8 \times 11 \times 19 h^{4}}{5 \times 9 !}-3 \frac{99509(2 h)^{6}}{4 \times 7 \times 11 !} \\
& \left.-2^{5} \frac{26030019779 h^{8}}{3 \times 5 \times 7^{2} \times 13 !}\right)+\cdots, \\
& g(h)=-\frac{h^{2}}{3 !}-\frac{17 h^{4}}{3 \times 5 !}+\frac{1417 h^{6}}{3 \times 7 !}-\frac{31319 h^{8}}{9 !} \\
& -3 \frac{3843187 h^{10}}{11 !}+3 \frac{1957250863 h^{12}}{13 !} \\
& -\frac{3995485914943 h^{14}}{3 \times 15 !}+\cdots, \\
& y(h)=4 h^{4}\left(4 \frac{11}{3 \times 5 !}-\frac{863 h^{2}}{3 \times 7 !}\right. \\
& -2^{4} \frac{67 \times 89 h^{4}}{5 \times 9 !}-\frac{39503543 h^{6}}{7 \times 11 !} \\
& \left.+2^{2} \times 11 \frac{15939718901 h^{8}}{3 \times 5 \times 7^{2} \times 13 !}\right)+\cdots .
\end{aligned}
$$

[1] M. J. Ablowitz and J. F. Ladik, J. Math. Phys. 16, 598 (1975).

[2] M. J. Ablowitz and J. F. Ladik, J. Math. Phys. 17, 1011 (1976).

[3] M. J. Ablowitz and J. F. Ladik, Stud. Appl. Math. 55, 213 (1976).

[4] M. J. Ablowitz and J. F. Ladik, Stud. Appl. Math. 57, 1 (1977).

[5] V. M. Kenkre and G. P. Tsironis, Phys. Rev. B 35, 1473 (1987).

[6] A. R. Its, A. G. Izergin, V. E. Korepin, and N. A. Slavnov, Phys. Rev. Lett. 70, 1704 (1993).

[7] D. Cai, A. R. Bishop, and N. Gronbech-Jensen, Phys. Rev. Lett. 74, 1186 (1995).

[8] A. A. Vakhnenko and Yu. B. Gaididei, Teor. Mat. Fiz. 68, 350 (1986) [Theor. Math. Phys. 68, 873 (1986)].

[9] A. B. Aceves, C. De Angelis, T. Peschel, R. Muschall, F. Lederer, S. Trillo, and S. Wabnitz, Phys. Rev. E 53, 1172 (1996).

[10] P. Marquie, J. M. Bilbault, and M. Remoissenet, Phys. Rev. E 51, 6127 (1995).

[11] J. M. Bilbault and P. Marquie, Phys. Rev. E 53, 5403 (1996).

[12] D. Hennig and G. P. Tsironis, Phys. Rep. 307, 333 (1999).

[13] V. E. Vekslerchik and V. Konotop, Inverse Probl. 8, 889 (1992).

[14] K. Narita, J. Phys. Soc. Jpn. 59, 3528 (1990).
[15] K. Maruno and Y. Ohta, J. Phys. Soc. Jpn. 75, 054002 (2006).

[16] V. E. Vekslerchik, J. Nonlin. Math. Phys. 9, 157 (2002).

[17] Q. Zhenyun, J. Math. Phys. 49, 063505 (2008).

[18] X. Liu and Y. Zeng, J. Phys. A 40, 8765 (2007).

[19] A. Ankiewicz, N. Devine, M. Ünal, A. Chowdury, and N. Akhmediev, J. Opt. 15, 064008 (2013).

[20] N. Akhmediev and A. Ankiewicz, Phys. Rev. E 83, 046603 (2011).

[21] A. Ankiewicz, N. Akhmediev, and F. Lederer, Phys. Rev. E 83, 056602 (2011).

[22] K. W. Chow, R. Conte, and N. Xu, Phys. Lett. A 349, 422 (2006).

[23] A. Ankiewicz, N. Akhmediev, and J. M. Soto-Crespo, Phys. Rev. E 82, 026602 (2010).

[24] A. Khare and A. Saxena, Pramana 78, 187 (2012).

[25] A. Khare, A. Saxena, and A. Khare, Pramana 79, 377 (2012).

[26] N. Akhmediev and A. Ankiewicz, Phys. Rev. Lett. 82, 2661 (1999).

[27] N. Akhmediev and A. Ankiewicz, Solitons, Nonlinear Pulses and Beams (Chapman \& Hall, London, 1997). 
[28] C. M. Schober, Phys. Lett. A 259, 140 (1999).

[29] M. J. Ablowitz and B. Prinari, Discrete and Continuous Nonlinear Schrödinger Systems (Cambridge University Press, Cambridge, 2004).

[30] A. Ankiewicz, W. Królikowski, and N. N. Akhmediev, Phys. Rev. E 59, 6079 (1999).

[31] A. Ankiewicz, Opt. Acta 25, 361 (1978).

[32] N. Akhmediev, W. Królikowski, and A. W. Snyder, Phys. Rev. Lett. 81, 4632 (1998).

[33] A. Hasegawa, Phys. Fluids 20, 2155 (1977).

[34] A. Hasegawa, Phys. Fluids 18, 77 (1975).

[35] A. Hasegawa, Opt. Lett. 5, 416 (1980).

[36] M. Mitchell, Z. Chen, M. Shih, and M. Segev, Phys. Rev. Lett. 77, 490 (1996).

[37] M. Segev and D. N. Christodoulides, Opt. Photon. News 13, 70 (2002).

[38] G. Duree et al., Phys. Rev. Lett. 71, 533 (1993); M. Shih et al., Electron. Lett. 31, 826 (1995).

[39] M. D. Iturbe-Castillo et al., Appl. Phys. Lett. 64, 408 (1994); A. A. Zozulya et al., Europhys. Lett. 36, 419 (1996); A. V. Mamaev et al., Phys. Rev. A 54, 870 (1996).
[40] D. N. Christodoulides, T. H. Coskun, M. Mitchell, and M. Segev, Phys. Rev. Lett. 78, 646 (1997).

[41] M. Mitchell, M. Segev, T. Coskun, and D. N. Christodoulides, Phys. Rev. Lett. 79, 4990 (1997).

[42] D. N. Christodoulides, T. H. Coskun, and R. I. Joseph, Opt. Lett. 22, 1080 (1997).

[43] A. W. Snyder and D. J. Mitchell, Phys. Rev. Lett. 80, 1422 (1998)

[44] V. V. Shkunov and D. Z. Anderson, Phys. Rev. Lett. 81, 2683 (1998).

[45] V. A. Vysloukh, V. Kuznetsov, V. M. Petnikova, and V. V. Shuvalov, Sov. J. Quantum Electron. 27, 843 (1997); V. Kutuzov, V. M. Petnikova, V. V. Shuvalov, and V. A. Vysloukh, Phys. Rev. E 57, 6056 (1998).

[46] I. Kay and H. E. Moses, J. Appl. Phys. 27, 1503 (1956).

[47] F. T. Hioe, Phys. Rev. E 58, 6700 (1998).

[48] O. O. Vakhnenko, Phys. Rev. E 64, 067601 (2001).

[49] O. O. Vakhnenko, Phys. Rev. E 60, R2492 (1999).

[50] O. O. Vakhnenko et al., Phys. Rev. E 61, 7110 (2000).

[51] A. Bülow, D. Hennig, and H. Gabriel, Phys. Rev. E 59, 2380 (1999). 